\title{
Fluid dynamics in crystal growth: The good, the bad, and the ugly
}

\author{
Jeffrey J. Derby \\ Department of Chemical Engineering and Materials Science, University of Minnesota, \\ 151 Amundson Hall, 421 Washington Avenue SE, Minneapolis, MN 55455-0132, USA
}

\begin{abstract}
Fluid dynamics are important in processes that grow large crystals from a liquid phase. This chapter presents a primer on fluid mechanics and convection, followed by a discussion of the physics and scaling of flows in such processes. Specific examples of fluid flows in crystal growth systems are presented and classified according to their impact on outcomes, good or bad. Turbulence in crystal growth is discussed within the limited extent of our understanding, which is incomplete, or ugly.
\end{abstract}

Keywords: Fluid mechanics, crystal growth, convection, segregation, instabilities

\section{Introduction}

This chapter is meant as an introduction toward understanding fluid dynamics and the effects that flow can bring about during the growth of large, single crystals. The title of this article is chosen in a shameless attempt to attract your attention by alluding to an epic film of the 1960s, directed by Sergio Leone and starring Clint Eastwood. However, this title also means to emphasize that flows in large-scale crystal growth systems are always important and that their effects can be beneficial (good) or detrimental (bad). Indeed, one motivation for their study is to understand their effects so that changes in process design or operation may produce better outcomes. What about the "ugly" in the title? Well, please continue reading to find out...

The literature on flows in crystal growth is vast, and no attempt will be made to present a comprehensive summary of it. Some recent reviews on this topic include those of Derby et al. [1], Kakimoto and Gao [2], Tsukada [3], Vizman [4], and Capper and Zharikov [5]. In this chapter, we will first focus on some essentials on flows and their effects, followed by a series of examples of interesting and important flows in crystal growth systems. We will focus on the growth of single crystals from liquids, with examples drawn from both

Email address: derby@umn.edu (Jeffrey J. Derby) 
solution and melt growth systems. We will not consider the many, interesting complications that may arise in vapor crystal growth processes [6] or during the crystallization of many crystals in purification operations [7].

\section{Background}

\subsection{A primer on fluid mechanics}

While fluid mechanics can become horrendously complicated, it is important to remember that fluids follow the same, basic kinematic rules that every scientist or engineer learned long ago, namely Newton's laws of motion. We write Newton's second law backwards (to make the next equation more clear) as,

$$
\begin{array}{ll}
m \mathbf{a} & =\mathbf{F}_{\text {net }}, \\
\text { mass } \times \text { acceleration } & \text { Net force }
\end{array}
$$

where the bold characters indicate vector quantities. This simple form is applicable to a rigid body. However, if we instead imagine a dollop of fluid upon which forces push and pull, we can carefully shrink it to an infinitesimal size and re-express the above expression in terms of the velocity of the fluid,

$$
\begin{array}{lll}
\rho\left(\frac{\partial \mathbf{v}}{\partial t}+\mathbf{v} \cdot \nabla \mathbf{v}\right)= & -\nabla p & +\mu \nabla^{2} \mathbf{v} \\
\begin{array}{l}
\text { mass per unit volume } \\
\times \text { acceleration }
\end{array} & \begin{array}{ll}
\text { pressure forces } \\
\text { per unit volume }
\end{array} & \begin{array}{l}
\text { viscous forces } \\
\text { per unit volume }
\end{array} \\
& +\rho_{0} \mathbf{g}\left[1-\beta\left(T-T_{0}\right)\right] & +\mathbf{F}(\mathbf{v}, \mathbf{x}, t), \\
& \begin{array}{l}
\text { buoyant force } \\
\text { per unit volume }
\end{array} & \begin{array}{l}
\text { applied body force } \\
\text { per unit volume }
\end{array}
\end{array}
$$

where the correspondence between eqs. (1) and (2) should be readily apparent. To define our nomenclature, $\rho$ is the density of the fluid, $\mathbf{v}$ is the velocity field, $t$ is time, $\nabla$ is the gradient operator representing derivatives over spatial dimensions $\mathbf{x}, p$ is the pressure field, $\mu$ is the fluid viscosity, $\mathbf{g}$ is the gravitational vector, $\beta$ is the thermal expansivity, $T$ is temperature, and the subscript zero denotes the reference state about which the linear dependence of fluid density on temperature is approximated.

The first two terms on the right-hand-side of eq. (2) represent different components of the net force acting among fluid elements. The pressure field, represented by the variable $p$, transmits forces acting normal to an element, while viscosity transmits forces via shear, i.e., momentum transferred by fluid sliding over adjacent elements. This expression arises from the work of Newton, who postulated that shear stresses are linearly proportional to velocity gradients. Fluids that obey this constitutive relation are referred to as Newtonian fluids.

The terms on the following line are body forces that act over the volume of the fluid. Gravity alone results in a hydrostatic pressure field that varies in 
elevation, whereas buoyant forces arise from gravity acting over density differences, represented here by the term involving $\beta\left(T-T_{0}\right)$. Buoyancy acts as a lever arm, whereby the net force arises from density differences perpendicular to the direction of gravity. The expression shown in eq. (2) arises from the Boussinesq approximation, which represents changes in density as a linear function of temperature (or composition, which is not shown above). The last term, $\mathbf{F}(\mathbf{v}, \mathbf{x}, t)$, is a catch-all for additional body forces that may act on the fluid. Some particularly useful outcomes can arise from forces of this type, such as Lorentz effects from the application of a magnetic field to a conducting fluid.

In the process of derivation of the previous equation, we made an additional, important assumption that the fluid itself is incompressible, i.e., that its density does not change appreciably with pressure, an assumption that is extremely good for a liquid and sometimes reasonable for a gas. While the above application of Newton's second law to a fluid manifests itself in the conservation of momentum, we must specify an additional constraint to guarantee continuity, i.e., the conservation of mass. This is written for an incompressible fluid as,

$$
\nabla \cdot \mathbf{v}=0
$$

Collectively, eqns. (2) and (3) constitute the celebrated Navier-Stokes equations.

\subsection{Convection - the effects of fluid flow}

A flowing liquid typically has a significant effect on local temperature and composition via convection, or advection in some fields. Convection is the transport of heat (thermal energy) and material (species) by flow. This is readily seen in the conservation equations derived for the temperature,

$$
\begin{array}{lll}
\rho C_{p} \frac{\partial T}{\partial t} & +\rho C_{p} \mathbf{v} \cdot \nabla T & =\kappa \nabla^{2} T, \\
\begin{array}{l}
\text { accumulation of } \\
\text { thermal energy } \\
\text { per unit volume }
\end{array} & \begin{array}{l}
\text { convective transport } \\
\text { of thermal energy } \\
\text { through unit volume }
\end{array} & \begin{array}{l}
\text { conductive transport } \\
\text { of thermal energy } \\
\text { through unit volume }
\end{array}
\end{array}
$$

where $C_{p}$ is the heat capacity and $\kappa$ is the thermal conductivity of the fluid. A more extensive discussion of heat transfer in melt crystal growth is provided in [8]. A similar equation is written for species conservation,

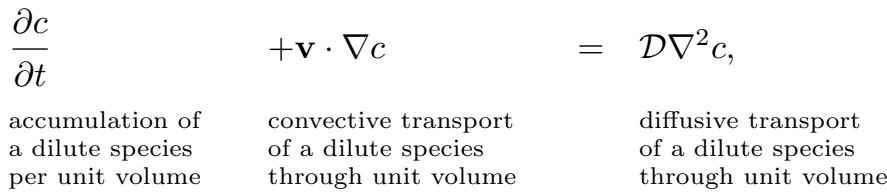

where $c$ denotes species concentration and $\mathcal{D}$ is the diffusion coefficient of the species in the fluid.

Fluid dynamics in crystal growth systems are most often important not because of the momentum carried by the flow but because of the effects of convection. Specifically, fluid flows modify heat and species transport in extremely important ways, and several examples will be discussed later in this chapter. 


\subsection{Understanding through scaling}

Finding analytical solutions to the Navier-Stokes equations is extremely challenging, and such solutions are generally available only for very simple systems [9]. Therefore, a useful approach for obtaining insight without solving the problem completely is approximating the relative magnitude of driving forces by appropriate scaling of the equations. The basic idea behind scaling analysis is to choose characteristic scales for length, velocity, time, etc., so that the governing equations are made dimensionless. The resulting groups of physical properties and characteristic scales form dimensionless numbers that can represent meaningful ratios of competing effects.

In the ensuing discussion, we will touch upon the few most important dimensionless groups for understanding flows in melt and solution crystal growth. For a more extensive discussion of dimensionless groups in crystal growth, the interested reader is referred to the still-relevant review by Brown. [10] and to many prior analyses that employ scaling for understanding such systems [11-18].

The single most important dimensionless group for understanding fluid flows is the heralded Reynolds number, defined as $R e \equiv V L / \nu$, where $V$ is a characteristic velocity, $L$ is a characteristic length, and $\nu \equiv \mu / \rho$ is the kinematic viscosity of the fluid. This dimensionless group arises naturally when the momentum balance, eq. (2), is re-written in dimensionless form using scalings of $\tilde{\mathbf{v}} \equiv \mathbf{v} / V$ to define a nondimensional velocity and $\tilde{\mathbf{x}} \equiv \mathbf{x} / L$ to define nondimensional spatial coordinates, where the tilde refers to the nondimensional variable.

The dimensionless form of the momentum balance is then given as,

$$
\frac{\partial \tilde{\mathbf{v}}}{\partial \tilde{t}}+\operatorname{Re}(\tilde{\mathbf{v}} \cdot \nabla \tilde{\mathbf{v}})=-\nabla \tilde{\mathcal{P}}+\nabla^{2} \tilde{\mathbf{v}}-\operatorname{Ra}\left(\tilde{T} \mathbf{e}_{g}\right)
$$

It is convenient to employ the dynamic pressure, $\mathcal{P} \equiv p-\rho g z$, that, via its definition, contains the hydrostatic term of the body force to simplify this equation. Note that the resultant scalings for time and pressure are $\tilde{t} \equiv t \nu / L^{2}$ and $\tilde{\mathcal{P}} \equiv \mathcal{P} L / \nu V$, respectively. Finally, we have also neglected the catch-all term for applied body forces that was specified in eq. (2).

We find that the last term, representing thermal buoyant forces, includes another dimensionless group, the Rayleigh number, defined as $R a \equiv g \beta \Delta T L^{3} / \nu \alpha$, multiplying a dimensionless temperature difference, $\tilde{T} \equiv\left(T-T_{0}\right) / \Delta T$, and $\mathbf{e}_{g}$, a unit vector in the direction of gravity. In these definitions, the thermal diffusivity is defined as $\alpha \equiv k / \rho C_{p}$ and we need to specify another scale, $\Delta T$, a characteristic temperature difference across the fluid. Alternatively, one may encounter the Grashof number, $G r \equiv g \beta \Delta T L^{3} / \nu^{2}$, in the buoyancy term of the dimensionless momentum equation. The Grashof number arises when one defines a characteristic time based on the thermal diffusion scaling of $L^{2} / \alpha$ rather than the viscous time scale of $L^{2} / \nu$, as applied in eq. (6). The Rayleigh number is related to the Grashof number by $\operatorname{Ra}=\operatorname{Pr} G r$, where $\operatorname{Pr} \equiv \nu / \alpha$ is the Prandtl number of the fluid.

When properly defined, these dimensionless numbers provide significant insight to the nature of the flow. The Reynolds number provides a measure of the 
ratio of inertial to viscous forces. The Reynolds number is always substantial for flows in large-scale crystal growth systems and represents the importance of the nonlinear effects of inertia. Systems with moderate Reynolds numbers, typically less than several thousands, usually exhibit laminar, time-independent flows. However, stronger flows bring more complicated behavior, and flows with Re larger than several thousands will almost always exhibit time-dependent behavior. As the Reynolds number increases further, the flow evolves toward turbulence and exhibits strong fluctuations in time and space.

Flows are driven by thermal buoyancy in melt crystal growth systems, and the Rayleigh and Grashof numbers are most useful for representing the strength of buoyant driving forces and the intensity of the resulting flows. ${ }^{1}$ The Rayleigh and Grashof numbers are linearly proportional to $\Delta T$, the temperature difference across the system, and scale with the cubed power of $L$, the characteristic dimension. This $L^{3}$ scaling indicates that buoyancy-driven flows grow geometrically with system size; convection is always much more intense in large-scale systems. The most appropriate measures for $\Delta T$ and $L$ are those lying in the horizontal plane, perpendicular to the force of gravity.

For most melt crystal growth processes, both the Reynolds and Rayleigh numbers are large. For a steady-state flow in such a system, we can ignore all terms of dimensionless eq. (6), except those representing the dominant forces of inertia and buoyancy to write,

$$
\operatorname{Re}(\tilde{\mathbf{v}} \cdot \nabla \tilde{\mathbf{v}}) \sim \operatorname{Ra} \tilde{T} .
$$

We argue that our scaling makes $\tilde{T} \approx 1$ and rearrange the balance to read,

$$
\tilde{\mathbf{v}} \cdot \nabla \tilde{\mathbf{v}} \sim R a / R e .
$$

We then perform a bit of algebra on both sides of this equation, during which we specify the characteristic velocity using the thermal diffusion time scale, $V \equiv \alpha / L$, and rewrite the left-hand side using a new Reynolds number based on the maximum velocity in the system, $R e^{*} \equiv v_{\max } L / \nu$. We finally arrive at a useful scaling ${ }^{2}$,

$$
\operatorname{Re}^{*} \sim G r^{1 / 2}
$$

that should describe strong, buoyant flows. Simulations have shown this relationship to be valid for many melt crystal growth systems.

We quickly mention that the heat and mass transfer equations, eqs. (4) and (5), can also be nondimensionalized to obtain groups that measure the ratio of convective to diffusive transport. We derive the dimensionless Peclet number, $P e \equiv V L / \alpha$, to measure the importance of convective heat transfer, and

\footnotetext{
${ }^{1}$ While not discussed here, Marangoni flows can also be quite important in systems with a melt-gas free surface. These flows arise from gradients in surface tension across the free surface and can be very strong; see, e.g., [3, 19, 20].

${ }^{2}$ There is a dimensionless geometric ratio, $L / \Delta L$, that arises from the approximation of $\nabla$ in eq. (8); however, we have ignored it in eq. (9), since it is constant for any specific system.
} 
a corresponding solutal Peclet number, $P e_{s} \equiv V L / \mathcal{D}$, to assess convective effects on mass transfer. Both of these numbers can also be expressed in terms of the Reynolds number, with $P e=R e P r$ and $P e_{s}=R e S c$, where the dimensionless Prandtl number, $\operatorname{Pr} \equiv \nu / \alpha$, and Schmidt number, $S c \equiv \nu / \mathcal{D}$, are defined by the physical properties of the fluid.

Before continuing to our discussion of specific examples of fluid dynamical effects in crystal growth systems, we desire to make one more point on the role and meaning of dimensionless numbers. In search of simple answers, many come to the false conclusion that discrete transitions in behavior are marked by universal, critical values of dimensionless numbers. For example, it is well established that flow in a pipe will be steady and laminar when its Reynolds number is less than 2,300 and will be time-dependent and turbulent when the Reynolds number exceeds 4,000 or so [21]. Notwithstanding this profound observation, the critical Reynolds number for turbulence is different for every geometry. Similarly, the onset of natural convective flow in the Rayleigh-Bénard configuration $[22,23]$, consisting of a fluid heated from below, occurs at a critical Rayleigh number of $R a_{c}=1,708$. However, this analysis only holds for the case of a fluid layer between infinite, parallel plates that are isothermal and perfectly horizontal. Though referred to time and time again in crystal growth literature, this classical problem for natural convection, including its well-known $R a_{c}$, has little relevance for real systems of finite extent with complicated heating. Thus, one must accept the more nuanced interpretation that dimensionless numbers are relative measures only; their precise interpretation is possible only in welldefined problems with simple geometries and boundary conditions. In crystal growth, the real behavior is strongly dependent upon the details of each system.

In summary, scaling is indispensable for mastering a mechanistic understanding of fluid flow. Nevertheless, the detailed knowledge of flows within a particular crystal growth system requires a direct solution of the governing equations, usually obtained via computational methods. The two approaches work best when applied together. We proceed in this vein with specific examples of flows in crystal growth systems.

\section{The Good}

Flows in crystal growth systems often bring about beneficial outcomes, which we label as "good." While there are many, many examples of "good" fluid dynamical behaviors, we can only present a few here, due to length limitations of this chapter. We draw upon two examples based on our prior research.

\subsection{Increasing growth rates in solution crystal growth via applied rotation}

Solution crystal growth systems employ a liquid solvent in which the crystallizing species is dissolved, followed by some means of bringing the liquid phase to a thermodynamically unstable state. Then the supersaturated species migrates from the liquid and precipitates onto a growing crystal. Achieving and maintaining supersaturation in the liquid phase to promote the growth of a large, single crystal is typically accomplished by a slow change in system temperature. 

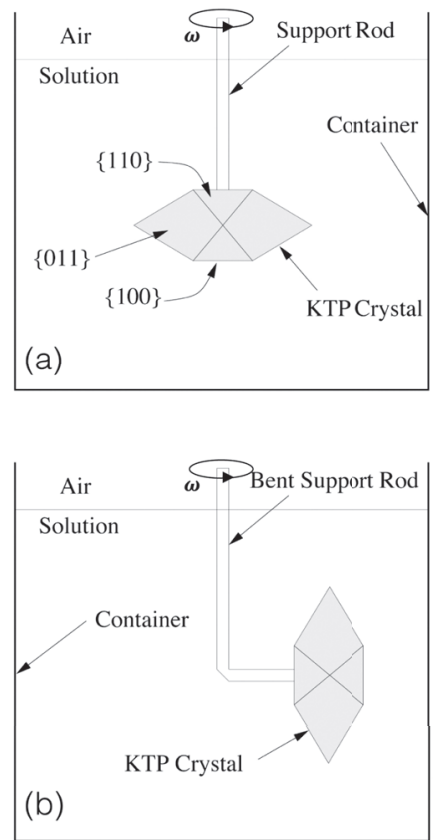

(c)

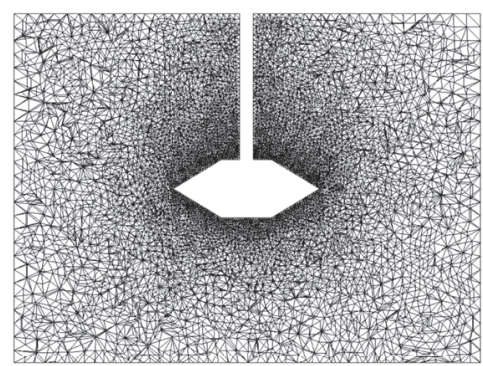

(d)

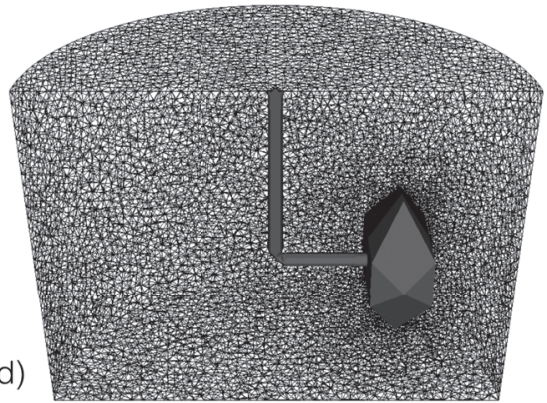

Figure 1: Schematic representations of KTP solution growth: (a) $0^{\circ}$ model and (b) $90^{\circ}$ model. Unstructured tetrahedral finite element meshes for these cases are depicted in (c) and (d).

For solution growth systems, severe limitations to the overall growth rate arise from the inherently slow diffusion of the solute molecule through the liquid solvent. Such limitations may be overcome by the suitable application of fluid flows that promote mass transfer of the solute via convection. Much experimental work has been devoted to this topic [24-33], and we have carried out extensive modeling studies of solution crystal growth systems [34-44].

We consider the specific example of the solution growth of potassium titanyl phosphate (KTP). The KTP growth system developed by Bordui and co-workers [30-32] presents an ideal candidate for study, particularly since the comparatively small size of the KTP crystal and high viscosity of the $\mathrm{K}_{6} \mathrm{P}_{4} \mathrm{O}_{13}$ solution from which it is grown results in laminar flows that are amenable to analysis. In this process, the KTP seed crystal is mounted directly to a support rod and fully immersed within the solution phase; the container and contents are enclosed within an isothermal zone maintained by heat pipes.

We consider the two extreme crystal mounting orientations mentioned in [31, 32 , namely the $0^{\circ}$ and the $90^{\circ}$ orientations, which are shown schematically in Figure 1, along with the finite element meshes employed to compute threedimensional flows in this system [37]. We consider flows driven by steady crystal rotation at $50 \mathrm{rpm}$ and ignore buoyant driving forces, which are insignificant here. For crystal of length $L=2.5 \mathrm{~cm}$ in this system, the Reynolds number is $R e=\omega L^{2} / \nu=252$, and the flows are steady and laminar. Physical properties 


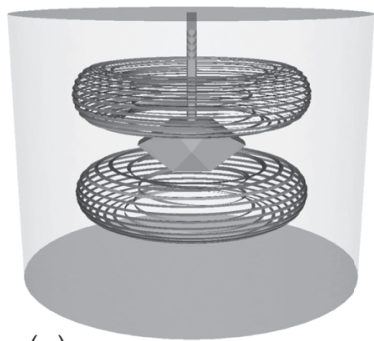

(a)

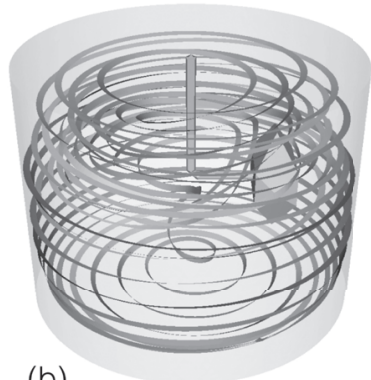

(b)

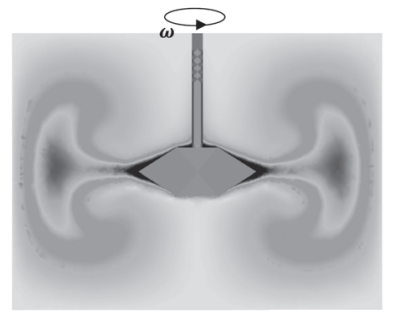

(c)

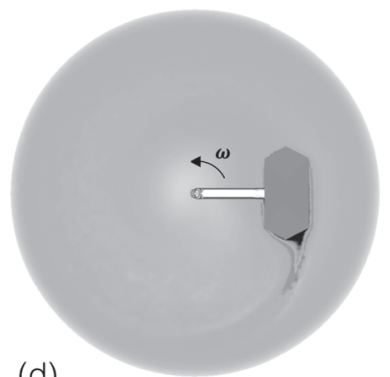

(d)

Figure 2: Pathlines of fluid motion driven by steady rotation of the crystal for (a) the $0^{\circ}$ case and (b) the $90^{\circ}$ case. Thin, solute depletion layers form near the crystal, as shown by supersaturation (lighter is higher and darker is lower) plotted (c) on a meridional plane for the $0^{\circ}$ case and (d) on an equatorial plane for the $90^{\circ}$ case.

are presented in [37], along with other details of the calculation.

Figures 2(a) and (b) depict the flows via pathlines that trace representative fluid elements in the systems. The strongest flows are azimuthal and driven by the rotating crystals; however, radial pressure gradients arise from centrifugal forces and drive secondary flows in the meridional planes. For the $0^{\circ}$ orientation, the flows are nearly axisymmetric, with rotation driving fluid outward along the upper and lower surfaces of the crystal. These flows turn as they approach the walls of the container and return via continuity to form torroidal vortices above and below the crystal. Secondary flows are significantly different in the $90^{\circ}$ orientation. For this configuration, high pressures are created in front of the leading facets of the crystal and a low pressure region is created in the trailing wake. The resulting flow around the crystal continuously disrupts any underlying axisymmetry, as evidenced by the pathline for this case, which originates from the same starting position as the lower path line in Figures 2(a) and is integrated for an identical length of time.

We are interested in the effects of these flows on solute transport to the crystal, which would be severely limited by bulk diffusive resistance in a quiescent liquid. We analyze the solute concentration, expressed here as the supersaturation, by solving the mass transport equation, eq. (5), assuming that growth at the crystal facets occurs via a first-order crystallization reaction. In both of these geometries, convection is of paramount importance (the solutal Peclet 
number is very large, $P e_{s} \approx 10^{7}$ ), and the details of the three-dimensional flows around the crystal set the pattern of supersaturation along the crystal surface and in the bulk.

The supersaturation level on a meridional plane cut through the $0^{\circ}$ model domain is shown in Figure 2(c). The effects of the secondary flows are evident from the similarity in structure between the supersaturation field and the toroidal flows shown in Figure 2(a). Thin layers of solution near the crystal surface are solute-poor from the first-order crystallization reaction occurring there, and these depletion layers are driven by convection outwards towards the container walls in the form of plumes. Axial flows move solute-rich solution towards the top and bottom $\{100\}$ facets of the crystal from regions of higher bulk supersaturation.

Flows in the $90^{\circ}$ configuration result in two dramatically different effects on the supersaturation field. First, the more complete kinematic mixing in the $90^{\circ}$ case (as evidenced by the flow pathline) leads to more uniform levels of supersaturation in the bulk of the fluid, as shown in Figure 2(d). Second, the pattern of supersaturation near the crystal is completely different from that produced in the $0^{\circ}$ configuration. Since the crystal is offset from the rotation axis, the $\{100\}$ facets are nearly aligned with the direction of the azimuthal flow, and fluid streams across these faces of the crystal. The flow collapses around the trailing faces of the crystal, and recirculating eddies over the outer trailing $\{110\}$ facet are closed, which leads to the depletion of solute in the wake region.

The overall outcome of fluid dynamics in both of these cases is "good," notably a very beneficial increase in solute transport to the crystal faces from the bulk solution, leading to much faster growth rates. From a mechanistic viewpoint, flows sweeping across the crystal faces dramatically thin the solute depletion layers caused by growth. Diffusion across these thin layers occurs far more readily than if were they absent. Further details of the flows in these two, different geometries will be discussed later in this chapter.

\subsection{Modifying interface shape in melt growth via a traveling magnetic field}

The application of an external magnetic field over an electrically conducting melt has long been of interest in crystal growth. The Lorentz forces that are produced under such fields can alter the form and strength of underlying flows; see, e.g., the recent review by Vizman [4]. Here, we are interested in a particular form for the magnetic field, in which an alternating current is phased through individual coils surrounding an crucible to produce a time-dependent, traveling magnetic field (TMF). The time-averaged outcome of this field is a Lorentz force acting in the meridional plane that can be varied in direction and magnitude to affect flows and heat transfer during Bridgman crystal growth [45].

Ono and Trapaga [46] were among the first to analyze TMF by modeling time-dependent magnetic induction in Czochralski growth. Long before, however, similar models had been developed for induction heating [47, 48], where it was desired to compute Joule heating rather than Lorentz force. Since then several studies have been published on TMF in vertical gradient freeze (VGF) or vertical Bridgman systems [49-55]. Recent modeling work related to the 

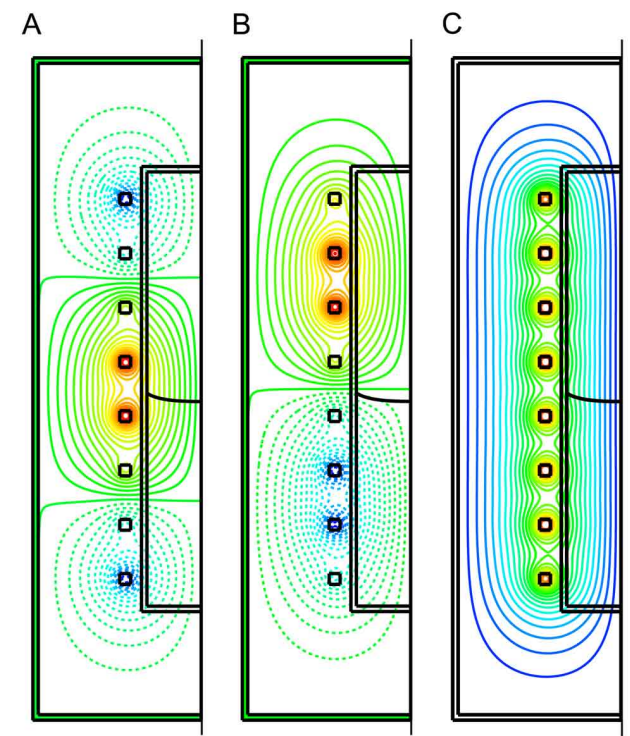

Figure 3: Model of a traveling magnetic field in a vertical gradient freeze crystal growth system, showing outer container, electric coils, and crucible containing melt above and crystal below. Geometry is cylindrical with centerline at right. (A) In-phase magnetic potential, $F$. (B) Out-of-phase magnetic potential, G. (C) Magnetic potential amplitude, $\|\mathbf{A}\|$. Contour spacing is $0.01 \mathrm{mT} \cdot \mathrm{m}$.

KRISTMAG project at the Leibniz Institute for Crystal Growth [56-64] is among the most detailed and integrated to date, though a decoupled approach has typically been used to solve for the magnetic field separately from the transport model. Yeckel and Derby [65] developed a self-consistent approach for modeling TMF applied to VGF systems and compared its effectiveness for control of interface shape in germanium and cadmium zinc telluride (CZT) crystal growth.

Here, we present sample results from [65] that illustrate outcomes for crystal growth that arise from fluid dynamics driven by TMF. The modeled system, illustrated in Figure 3, consists of a cylindrical, 4-inch-diameter VGF crucible surrounded by coils of an electrical conductor to which AC current is fed. A model of electrical induction based on Maxwell's equations is solved to determine the Lorentz force acting on the melt, which is coupled to a solidification model of convective heat transfer and phase change. More details are available in [65].

We first compute the components of a time-periodic, azimuthal magnetic potential, $A_{\theta}=F(r, z) \sin \omega t+G(r, z) \cos \omega t$, where $F$ and $G$ represent the amplitude of in-phase and out-of-phase components of the potential, respectively, with $\omega$ representing angular frequency, and from which the traveling magnetic field is obtained via $\mathbf{B}=\nabla \times \mathbf{A}$. Figures $3 \mathrm{~A}-\mathrm{C}$ show contours of the in-phase component $(F)$, out-of-phase component $(G)$, and amplitude $(\|\mathbf{A}\|)$ of the magnetic potential for a typical scenario, with an applied current $I_{o}=300 \mathrm{~A}$ and 

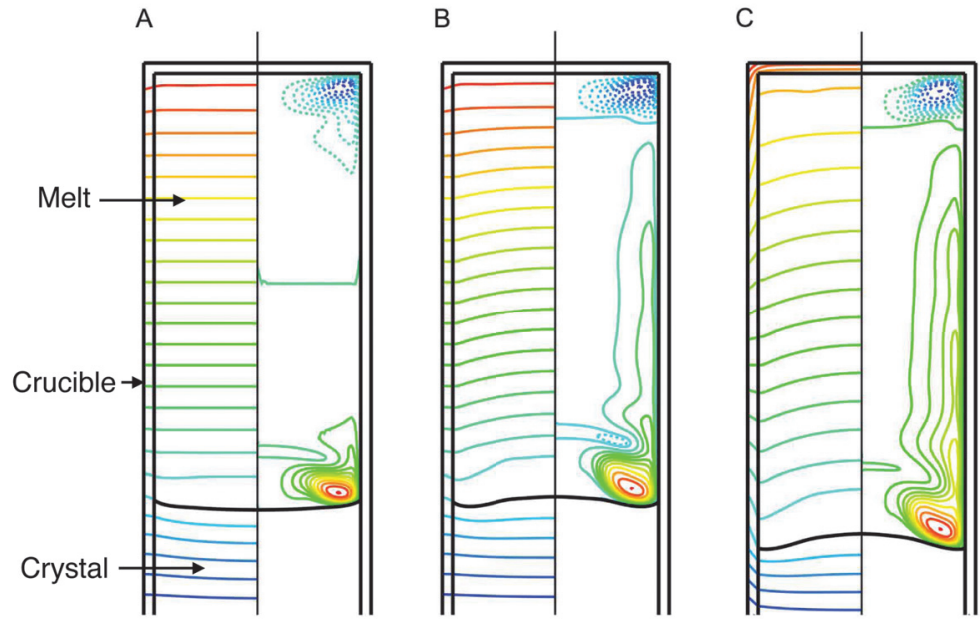

Figure 4: Temperature isotherms (left, plotted in $10 \mathrm{~K}$ intervals) and melt streamlines (right, solid lines indicate clockwise circulation, dotted lines counterclockwise) for steady-state germanium crystal growth. (A) Buoyancy alone, graphite crucible. (B) Buoyancy and TMF, graphite crucible. (C) Buoyancy and TMF, quartz crucible.

frequency $f=50 \mathrm{~Hz}$. By construction, the magnetic field direction is everywhere tangent to contours of the magnetic potential. The vertical traveling wavelike character of the potential can clearly be seen by the in-phase and outof-phase components, with a wavelength under these conditions approximately equal to the length of the crucible. The components of the oscillating magnetic field are then used to compute a time-averaged, net Lorentz force that acts in the meridional plane of the crystal growth melt.

Figure 4 demonstrates the beneficial effect that TMF can have on interface shape in germanium crystal growth. Under conditions of no applied magnetic field and steady-state growth conditions, shown in Figure 4A, the germanium crystal in a graphite crucible exhibits a concave interface shape. The flow near the melt-solid interface is driven upward at the centerline and downward along the crucible by buoyant forces arising from the radial temperature profile (which is slightly warmer at the center and cooler near the crucible wall). The Grashof number for this system is significant, $G r=3.6 \times 10^{8}$, so the flow is quite strong, with $R e=1,200$, based on the maximum melt velocity. However, its effect on heat transfer is only moderate, since $\operatorname{Pr}=0.016$ for germanium [66], yielding a Peclet number of $P e \approx 19$. Isotherms near the growth interface are only slightly deflected by convection in this case.

Figure 4B shows that adding TMF to this system notably changes the interface shape, inverting it from concave to convex over a large area around the center axis. This comes about from a significant strengthening of the buoyant flow cell near the interface. The time-averaged Lorentz force acts on the fluid in the meridional plane, pushing downward with more force near the crucible wall and decreasing in strength as the centerline is approached. This radial 
distribution of force is due to weakening of the magnetic field as it penetrates more deeply into the melt. Overall, there is a net force acting to increase the clockwise circulation of the vortex near the melt-solid interface. The stronger flow convects more heat downward toward the outer portions of the interface, while simultaneously acting to convect heat upward along the centerline (opposite to the direction of conduction). The outcome is a change of the concave interface shape to one that is convex across much of its extent.

Figure $4 \mathrm{C}$ shows that the convex region of the interface can be further extended by changing the crucible material from graphite to quartz. The quartz crucible does not shield the melt from the magnetic field as much as the electrically-conducting graphite crucible, so the strengthening of the vortex near the interface is even greater, convecting more heat toward the interface and driving it further downward while making it more convex. ${ }^{3}$ This is an example of a "good" flow driven by an applied field that provides an improved (convex) interface shape ${ }^{4}$.

Favorable reports on TMF have been made for the melt growth of Ge [57], GaAs [70], InP [50], GaInSn [54], and Si [61]. Initially there was optimism that TMF could also be used to improve cadmium zinc telluride (CZT), which is notoriously difficult to grow $[71,72]$. However, Yeckel and Derby [65] demonstrated that, even under large currents and high frequencies, TMF proves ineffectual for CZT growth. This outcome can be understood via scaling arguments, discussed more completely by Yeckel and Derby [65], showing that the ratio of Lorentz forces to buoyancy forces is represented by a dimensionless number known simply as the interaction parameter,

$$
\mathrm{N} \equiv \frac{\sigma \omega \mu^{2} I_{o}^{2}}{\rho g L \beta \Delta T}
$$

where $\sigma$ is electrical conductivity and $I_{o}$ is the applied current. A large interaction parameter is needed for TMF-driven flows to overpower the underlying natural convection and affect interface shape. For the growth of germanium considered here, $N=5$ and TMF proves to be effective. The interaction parameter for CZT growth under similar conditions is very small, $N=6.7 \times 10^{-3}$, and TMF does not significantly impact the system, unless unreasonably high currents are applied. To improve interface shapes in CZT growth, the design of more favorable furnace profiles is likely a far better approach than the application of TMF; see, e.g., the promising ideas put forth in $[8,73]$.

\footnotetext{
${ }^{3}$ Note that, for both of these cases, the shape of the interface contacting the crucible wall is not affected by melt convection, rather its shape is primarily determined by the mismatch in thermal conductivities between solid, melt, and crucible, as explained in the classical analysis of Jasinski and Witt [67]. See also the discussion in [8].

${ }^{4} \mathrm{An}$ interface shape that is convex with respect to the crystal has been postulated to minimize the potential for defects that may arise from deleterious ampoule wall interactions, such as dislocations, grains, or twins, to propagate toward the bulk crystal $[8,68,69]$.
} 


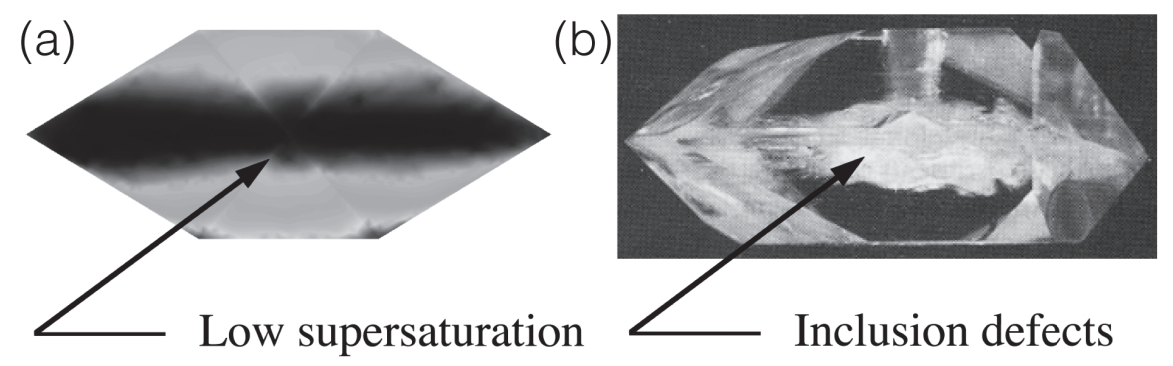

Figure 5: (a) Surface supersaturation predicted for the $0^{\circ}$ configuration; light denotes high supersaturation and dark denotes low supersaturation. (b) Inclusion defects on a KTP crystal grown using the $0^{\circ}$ configuration; adapted from [31, 37].

\section{The Bad}

Here, we present several interesting and important examples of "bad" flows, i.e., fluid dynamical effects that lead to unfavorable outcomes in crystal growth systems. As in the previous section, we limit ourselves to just a few examples from our prior research.

\subsection{Inclusion formation during solution crystal growth}

We return to the solution crystal growth system discussed previously to consider the possible deleterious effects of flows in these system. Fluid flows can promote undesirable morphological instabilities during growth which can lead to habit modification, macrostep formation, and liquid inclusions [24]. In particular, there is abundant experimental evidence demonstrating the important role of flow on inclusion formation $[28-32,74,75]$. There have also been many theoretical analyses of such morphological instabilities [39, 43, 76-84].

For the two KTP crystal mounting geometries shown in Figure 1, differences in solution flows strongly affected the supersaturation fields, as demonstrated in Figure 2. The resulting patterns of supersaturation on the surfaces of the crystals can be related to inclusion formation tendencies. Specifically, low levels of supersaturation occur along the equatorial regions of the crystal in the $0^{\circ}$ model; these regions occur due to the effects of local flow separation and recirculation. Significantly, experimental results of Bordui and Motakef [31] showed that the $0^{\circ}$ orientation resulted in many trapped liquid inclusions. Bordui and Motakef argued that these solute-poor regions would slow surface growth steps emanating from regions of high supersaturation, which would lead to step bunching and the onset of inclusion formation. Figure 5 compares our predictions of surface supersaturation with the pattern of inclusions in a typical KTP crystal grown using the $0^{\circ}$ orientation. The correspondence between the solute-poor region and the locations of inclusions is striking.

There are also regions of very low surface supersaturation for the steadilyrotated crystal in the $90^{\circ}$ orientation case. However, Bordui and co-workers 
$[31,32]$ found that growth using this geometry eliminated the formation of inclusions. The flow and mass transfer computations discussed in the previous section provide a mechanistic explanation for this outcome. Our results show that the local flows for the $90^{\circ}$ configuration result in a trailing wake structure with low surface supersaturations on the backward-facing facets of the crystal. Upon change in the direction of rotation, the flow and supersaturation pattern simply reverse from trailing to leading. In a time-averaged sense, periodic rotation reversal acts to homogenize the supersaturation field on the crystal surface, and the effects of time-averaging are likely to prevent the onset of inclusion formation in the $90^{\circ} \mathrm{KTP}$ growth configuration. This is not the case for the $0^{\circ}$ orientation, where the secondary flows and associated surface supersaturation patterns remain unchanged with the direction of crystal rotation. We more completely discuss the effects of rotation reversal via time-dependent simulations of this system in [40].

We showed previously that flows in solution crystal can be beneficial by increasing mass transfer and growth rate. However, we have shown here that inhomogeneity in mass transfer, caused by the details of three-dimensional flow patterns, can promote morphological instability and inclusion formation. These are definitely "bad" outcomes caused by fluid dynamics.

\subsection{Growth rate limits in the traveling heater method}

The traveling heater method (THM) is an important technique for producing compound semiconductor crystals, particularly detector-grade cadmium zinc telluride (CZT) [85-87]. Unlike the Bridgman method, the THM employs a liquid zone that is enriched in one component of the compound (tellurium, for the growth of CZT), which acts as a solvent. As the heater travels past the ampoule, it forms and moves this liquid zone to steadily dissolve a solid feed above while simultaneously growing a crystal of a similar composition at the opposite end of the zone. Since the crystal grows from a liquid flux, the THM allows for operation at much lower temperatures than the Bridgman process, and its path through the phase diagram results in comparatively better stoichiometry.

Despite the strengths of the THM, it is currently hampered by growth rates that are more than one order of magnitude slower than those routinely employed in Bridgman methods [71, 72, 85-89]. Prior models for this process have suggested that morphological instability could readily arise in THM [90, 91]. However, these past works did not identify the mechanisms that are responsible for such instability. Recent analysis by Peterson et al. [92] demonstrated that a classical fluid dynamical behavior explains this growth limitation.

This fluid flow gives rise to features commonly referred to as "lee waves," which are associated with atmospheric flows that oscillate within a vertically stratified fluid [93]. Such waves, depicted in Figure 6, typically arise in the lee of a mountain range where there is a vertically stabilized density field that arises from a cool mesa or valley below the flow. These structures are readily identified by cloud formations that form in the upper portion of the waves, which often result in spectacular, lenticular clouds. 

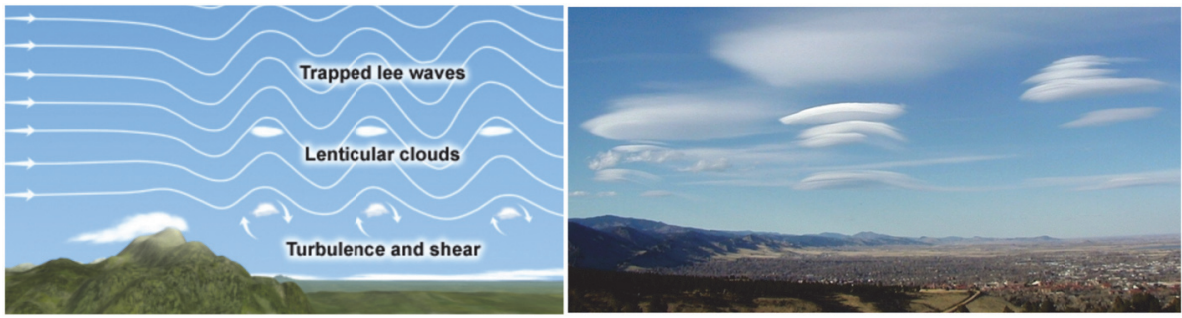

Figure 6: Left: A schematic diagram illustrating the formation of atmospheric lee waves. Adapted from the COMET Website, http://meted.ucar.edu. Right: Lenticular clouds that have resulted from lee waves. From Bill Randel, http://acd.ucar.edu/ randel/clouds.html

Lee waves come into being from an oscillatory response of the fluid after the flow has been vertically perturbed by a mountain. Consider a parcel of fluid and the vertical body forces acting upon it. Via Newton's second law, we can write a simple balance,

$$
\frac{\partial^{2} z^{\prime}}{\partial t^{2}}=\frac{g}{\rho_{0}} \frac{\partial \rho}{\partial z} z^{\prime}
$$

where $z^{\prime}$ is the with mean elevation of the fluid parcel, $z$ denotes vertical elevation, $g$ is the gravitational constant, and $\rho$ is the fluid density.

In a vertically stabilized density field, denser fluid lies under lighter fluid, so $\partial \rho / \partial z<0$. If we assume this value to be constant, we can obtain a solution to the above equation as,

$$
z^{\prime}=A \cos \omega t+B \sin \omega t
$$

where $A$ and $B$ are constants, indicating that the fluid parcel is oscillating vertically while traveling horizontally. This leads to waves with a frequency given by,

$$
\omega \equiv N=\sqrt{-\frac{g}{\rho_{0}} \frac{\partial \rho}{\partial z}}=\sqrt{g \beta \frac{\partial T}{\partial z}},
$$

where $N$ is known as the classical Brunt-Väisälä frequency [94] . The expression on the far right is written in terms of the vertical temperature gradient, $\partial T / \partial z$, and the Boussinesq approximation, with $\beta$ representing the thermal expansion coefficient of the fluid. Thus, the frequency of oscillation and the wavelength of the resulting flow structure depend on the magnitude of the vertical temperature gradient.

Let us now turn from the atmosphere to the THM system. Figure 7 shows simulations for the steady-state growth of a $3.2 \mathrm{~cm}$ diameter CdTe crystal via THM, where flow streamlines are plotted on the left and the temperature field is depicted on the right via isotherms. Adjacent streamlines and isotherms are plotted in constant increments. The CdTe solid feed is above and dissolving into the Te-enriched liquid zone, and a single crystal of CdTe grows downward at a rate of $1.24 \mathrm{~mm} /$ day from the zone. Simultaneous dissolution and growth are driven by the upward motion of the traveling heater (which is not shown 


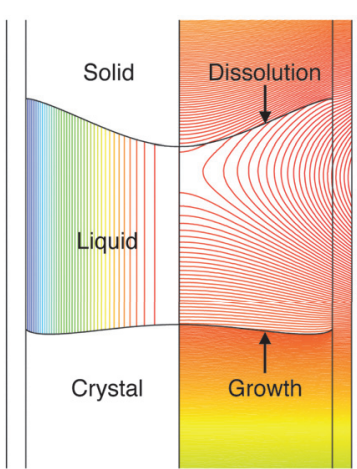

(a)

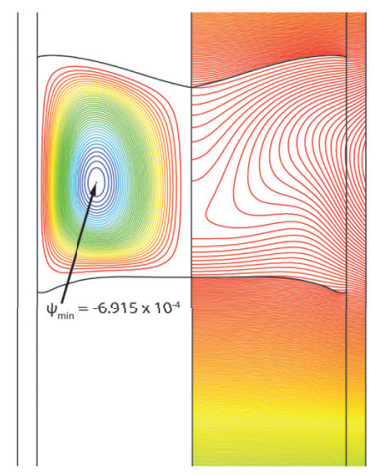

(b)

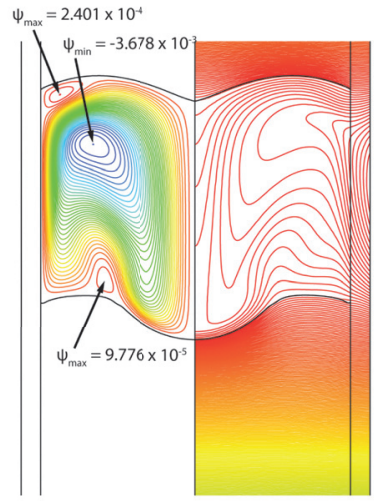

(c)

Figure 7: Model for steady-state THM crystal growth of CdTe, showing streamlines on the left and temperature contours on the right. (a) Without gravity. (b) With gravity set to $1 \%$ of earth. (c) With terrestrial gravity forces; note the formation of a lee wave structure with vortex adjacent to the growth interface.

in the figure). We consider how the flow in the zone evolves by changing the magnitude of the gravitational force in these cases.

Figure 7 (a) shows a calculation without gravity, namely with $g=0$. The streamlines on the left show a constant downward flow through the liquid zone caused by the change in density from solid to liquid; the spacing of the streamlines indicates the changing cross sectional area with radial position in the cylindrical zone. The zone is established by the surrounding heater, as reflected by the temperature isotherms on the right, showing hotter temperatures along the outer ampoule wall and heat diffusing into the liquid zone. The strongly coupled nature of this system is evident by the changing temperature across the interfaces, most readily seen along the upper, dissolution interface, which results from the composition-dependent melting temperature from the phase diagram.

The middle image, Figure $7(\mathrm{~b})$, is a calculation for gravity set to $1 \%$ of its terrestrial value. Here, a single, torroidal vortex occupies the liquid zone. Warmer, lighter fluid near the ampoule wall rises, while the cooler, denser fluid sinks along the centerline. This recirculating flow is fairly weak but still strong enough to slightly deflect the temperature isotherms, due to the fairly large Prandtl number for the liquid in the zone; $\operatorname{Pr} \approx 0.4$ is assumed for the liquid.

Under the effects of full gravity on earth, depicted in Figure 7(c), the flow is quite strong, with $G r \approx 1.5 \times 10^{6}$ and $R e \approx 400$. The primary vortex spins in the same direction as the prior case, directing a strong flow downward along the system centerline. As the liquid in the hot zone plunges toward the cooler growth interface, it feels a resistance from the stable vertical stratification of density. As the liquid turns to flow outward along the growth interface, the situation is analogous to the perturbed flow over the cool mountain valley, as depicted in Figure 6 , and a single lee wave forms with an accompanying, counter-rotating vortex. 


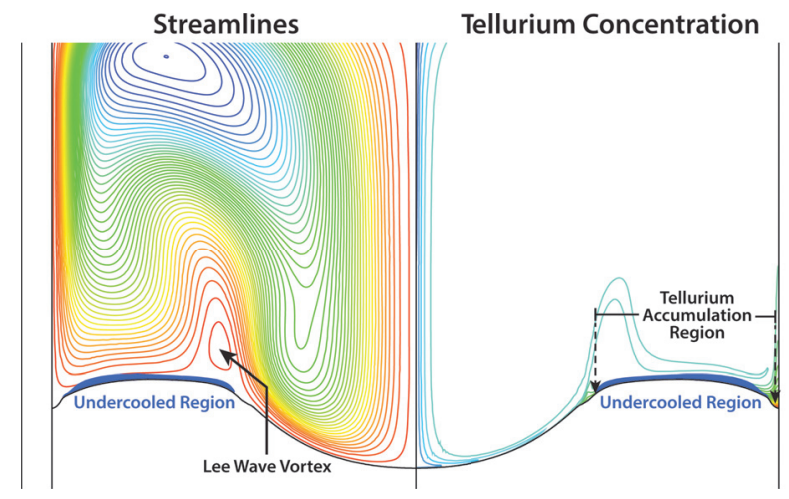

Figure 8: The formation of a lee wave vortex promotes the accumulation of excess tellurium in the liquid zone adjacent to the growth interface during the THM growth of CdTe. This accumulation leads to thermodynamically undercooled regions in the liquid adjacent to the growth interface, resulting in morphological instability and growth rate limitations.

The effect of this lee wave and vortex is shown in Figure 8, where flow streamlines are shown on the left and the tellurium iso-concentration contours are depicted on the right. The vigorous flows in the liquid promote mixing, and the Te concentration is nearly uniform in the bulk, with thin boundary layers exhibited along the centerline and growth interface. Due to segregation, excess tellurium is rejected from the growing solid and is subsequently swept outward along the interface and into the bulk as the flow turns upward along the inner ampoule wall. However, the counter-rotating vortex nested under the lee wave reverses the flow over a portion of the interface, creating a resistance to mass transport and promoting the local accumulation of tellurium. This accumulation leads to large concentration gradients within the solutal boundary layer. When these gradients are large enough, the composition-dependent melting point increases faster than the temperature field extending into the liquid. This results in an substantial region of supercooled liquid immediately in front of the growth interface, as shown in Figure 8, leading to constitutional supercooling and an unstable growth situation.

Significantly, the "bad" fluid dynamics in the THM liquid zone are the primary cause of the supercooling in this system, and this is quite different than the classical scenario of constitutional supercooling during melt crystal growth derived by Mullins and Sekerka [95]. In fact, due to the lee wave in the liquid zone, supercooling in this system occurs at growth rates more than an order of magnitude smaller than expected from such classical arguments. Paradoxically, the traditional strategy to alleviate Mullins-Sekerka constitutional supercooling during crystal growth, namely increasing the axial temperature gradient at the interface, is expected to strengthen the lee-wave vortex and accentuate the onset of instability. Thus, different strategies, that somehow lessen the impact of this fluid dynamical instability, will be needed to overcome growth limits in the traveling heater method. 


\section{The Ugly}

We finish this discussion of fluid dynamics in crystal growth with an examination of turbulence, of which our understanding is certainly incomplete, or "ugly." Turbulence arises when the nonlinear effects of inertia dominate over the damping effects of viscosity, and the transition from a laminar flow to a turbulent one is often characterized by a critical threshold in Reynolds number, as discussed previously. However, the detailed spatial and temporal characteristics of the zig-zagging, swirling vortices of a turbulent flow are poorly understood.

Historically, the classical scientific community has appreciated this challenge but has largely abandoned most of its attempts for deeper understanding. In an address to the British Association for the Advancement of Science in 1932, the acclaimed applied mathematician Horace Lamb stated, "I am an old man now, and when I die and go to heaven there are two matters on which I hope for enlightenment. One is quantum electrodynamics, and the other is the turbulent motion of fluids. And about the former I am rather optimistic." A giant in physics, nobel-prize winner Richard Feynman (who, coincidently, also made significant contributions to the understanding of quantum electrodynamics), stated in 1963 that "Turbulence is the most important unsolved problem of classical physics." Modern computational modeling of turbulence flows has furthered our understanding, but Moin and Kim [96] stated in 1997, "For a phenomenon that is literally ubiquitous, remarkably little of a quantitative nature is known about it." Even with nearly two decades of advances in computing power, our understanding of turbulence remains imperfect.

In melt crystal growth, turbulence arises in large-scale growth systems, particularly the Czochralski (CZ) growth and directional solidification (DS) of crystalline silicon, processes carried out in crucibles with dimensions of meters. With buoyant forces scaling with lateral dimension to the cube power, convective flows in these systems are orders of magnitude stronger than in the smaller-scale systems we have discussed above. Important outcomes of these turbulent flows are time-dependent variations in the crystal growth rate and compositional striations in grown crystals. These heterogeneities in composition lead to undesirable variations in material and electronic properties.

We will certainly not do justice to this complicated field; however, we wish to discuss the transition to turbulent flows under increasingly strong diving forces of buoyancy, which is of paramount importance for fluid flows in melt crystal growth systems. We turn to a series of landmark papers, in which Kim, Witt, and Gatos grew crystals of tellurium-doped indium antimonide [97] and gallium-doped germanium [98] using the vertical Bridgman method with the melt oriented beneath the crystal. This configuration is termed destabilizing, since it leads to an inherently unstable melt, with warm fluid underlying the cooler crystal, similar to the classical Rayleigh-Bénard problem [22, 23]. Since the crystal is growing with time, the height of the melt column is continuously decreasing and the driving force for convective flow is decreasing.

Samples of their results are shown in Figure 9, where axial sections of the grown, etched crystal are shown (revealing composition through changes in con- 


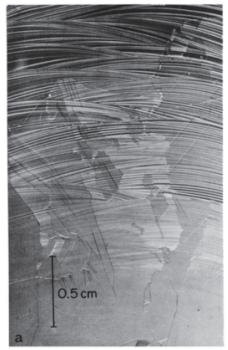

(a)

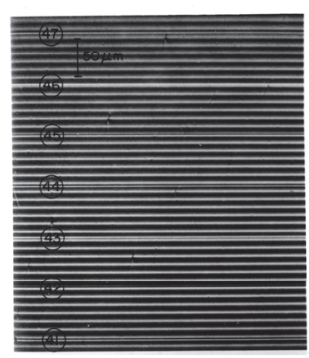

(c)
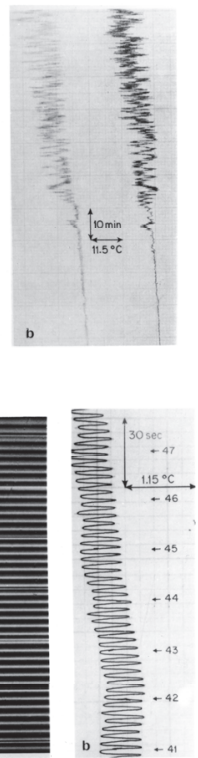

.

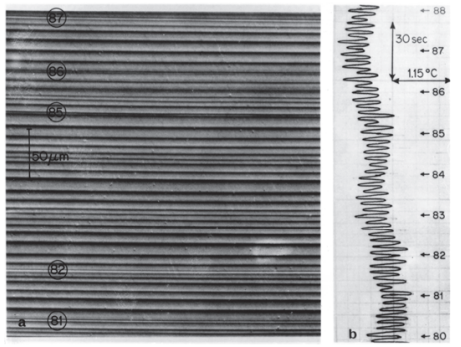

(b)

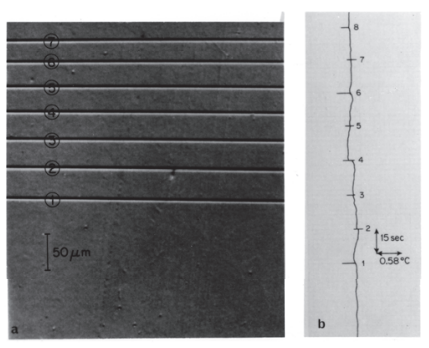

(d)

Figure 9: Etched crystal sections and melt thermocouple readings from the destabilized Bridgman growth of indium antimonide show transitions from (a) turbulent to (b),(c) transitional, time-dependent and time-periodic to (d) steady, laminar flows. Adapted from [97]

trast), along with traces obtained from a thermocouple placed in the melt at a uniform distance from the crystal interface. Figure 9(a) shows an axial section of the complete crystal (note the $0.5 \mathrm{~cm}$ scale bar). Irregular striation patterns and temperature readings are evident in the upper portion of the crystal, while the lower portion is much more uniform. Figures $9(\mathrm{~b})-(\mathrm{d})$ show greatly expanded views of the crystal (note the $50 \mu \mathrm{m}$ scale bars) and temperature traces at several growth intervals. A technique known as Peltier pulsing was used to demarcate the position of the melt-crystal interface at regular time intervals, indicated by the numbers in the figures. Kim et al. inferred from the striation patterns and temperature measurements that, as solidification progressed, the melt first exhibited turbulent convection, followed by successively less complicated oscillatory flows, followed by a steady, laminar flow. These fascinating flow transitions are a direct consequence of a reduction in flow intensity caused by the decreasing height of the melt.

Through these observations, Kim et al. elegantly demonstrated the importance of macroscopic processing conditions on establishing convective flow states which, in turn, profoundly affected the microscopic composition of melt-grown crystals. This fundamental observation also established the belief that a more complete understanding of fluid dynamics would be important for improving melt crystal growth processes.

We will end this chapter as we started, with another shameless allusion to a 

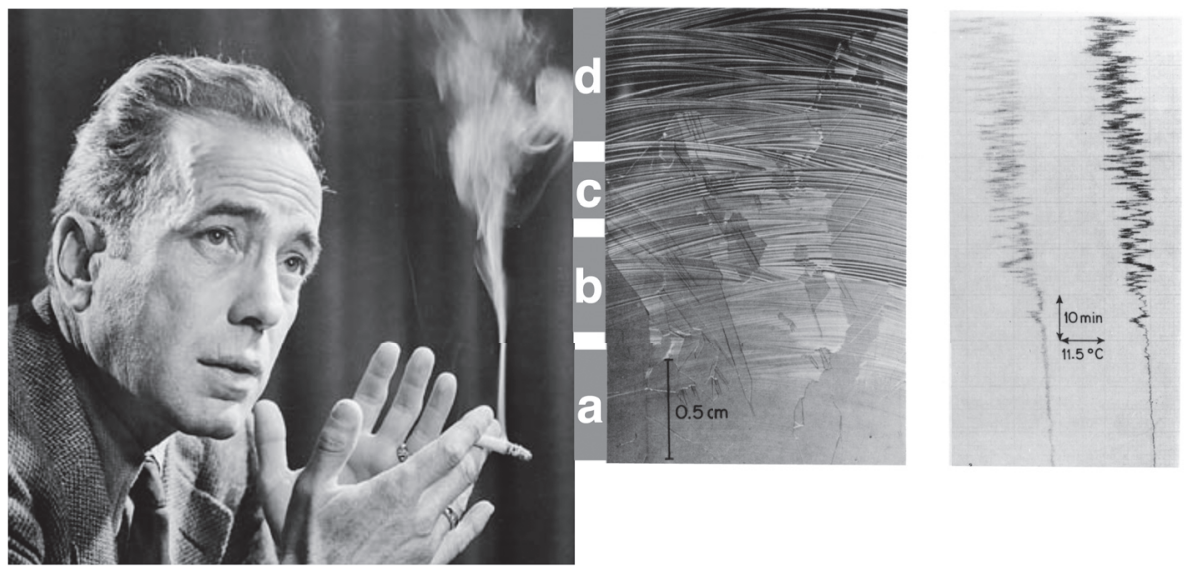

Figure 10: Buoyancy drives flows around a heated plume on the left and in melt crystal growth on the right (adapted from [97]). Flow transitions are driven by increasingly strong driving forces $(G r)$ with greater inertia $(R e)$. As both $G r$ and Re increase with height, these flows become: (a) laminar, (b) transitional, (c) weakly turbulent, and (d) strongly turbulent.

movie classic. Figure 10 shows a photograph of the legendary Humphrey Bogart along with the etched crystal grown by Kim, Witt, and Gatos (from the previous figure). This pairing of images demonstrates the correspondence between the physical behavior of buoyant fluid flows that evolve to turbulence in very different systems. The flows in both systems are driven by buoyancy, and the magnitude of the driving force increases with length scale, namely the height of Bogie's cigarette plume and the height of the melt in the destabilized Bridgman system (by the way, cigarettes and smoking are definitely ugly). Driving forces increase dramatically with length scale, and the flows associated with these forces evolve from steady and laminar, when the length scales are small, to erratic and turbulent, when length grows large. Finally, even though our detailed knowledge of turbulent flows remains "ugly," there is a certain beauty in our general understanding of the transitions leading to these flows.

\section{Final remarks}

Fluid flow is ubiquitous in all large-scale crystal growth systems. The effects of these flows can be good or bad, and our understanding is often incomplete and ugly. Progress is being made, and even turbulence is succumbing in degrees to persistent modeling refinements. On the whole, our understanding of fluid dynamics in crystal growth is expanding, and, particularly via the skillful application of modern computational modeling, continued progress and improved outcomes are certain. 


\section{Acknowledgments}

The author would like to acknowledge the support of many organizations, including current funding by the United States DHS, DOD, DOE, NASA, and NSF. The content of this paper does not necessarily reflect the position or policy of the United States Government, and no official endorsement should be inferred. The author would also like to acknowledge B. Vartak, J. Peterson, and A. Yeckel for specific content presented here, M. Divecha and K. Wang for feedback on this chapter, and all of his graduate students who have provided stimulus for expanding his understanding; however, all opinions and biases expressed in this chapter are his own.

[1] J. J. Derby, Modeling and bulk crystal growth processes: What is to be learned?, in: M. Wang, K. Tsukamoto, D. Wu (Eds.), Selected Topics on Crystal Growth: 14th International Summer School on Crystal Growth, Vol. 1270 of AIP Conference Proceedings, Melville, New York, 2010, pp. 221-246.

[2] K. Kakimoto, B. Gao, Fluid dynamics: Modeling and analysis, in: T. Nishinaga, P. Rudolph (Eds.), Handbook of Crystal Growth, 2nd Edition, Elsevier, Boston, 2015, pp. 845-870.

[3] T. Tsukada, The role of Marangoni convection in crystal growth, in: T. Nishinaga, P. Rudolph (Eds.), Handbook of Crystal Growth, 2nd Edition, Elsevier, Boston, 2015, pp. 871-907.

[4] D. Vizman, Flow control by magnetic fields during crystal growth from melt, in: T. Nishinaga, P. Rudolph (Eds.), Handbook of Crystal Growth, 2nd Edition, Elsevier, Boston, 2015, pp. 909-950.

[5] P. Capper, E. Zharikov, Oscillatory-driven fluid flow control during crystal growth from the melt, in: T. Nishinaga, P. Rudolph (Eds.), Handbook of Crystal Growth, 2nd Edition, Elsevier, Boston, 2015, pp. 951-993.

[6] R. Talalaev, Transport phenomena in vapor phase epitaxy reactors, in: T. F. Kuech (Ed.), Handbook of Crystal Growth, 2nd Edition, NorthHolland, Boston, 2015, pp. 909-942.

[7] D. A. Green, Crystallizer mixing: Understanding and modeling crystallizer mixing and suspension flow, in: A. S. Myerson (Ed.), Handbook of Industrial Crystallization, 2nd Edition, Butterworth-Heinemann, Boston, 2002, pp. $181-200$.

[8] J. J. Derby, A. Yeckel, Heat transfer analysis and design for bulk crystal growth: Perspectives on the Bridgman method, in: T. Nishinaga, P. Rudolph (Eds.), Handbook of Crystal Growth, 2nd Edition, Elsevier, 2015, pp. $793-843$.

[9] C. Y. Wang, Exact solutions of the steady-state Navier-Stokes equations, Ann. Rev. Fluid Mech. 23 (1991) 159-177. 
[10] R. A. Brown, Theory of transport processes in single crystal growth from the melt, AIChE Journal 34 (6) (1988) 881-911.

[11] F. Rosenberger, Fundamentals of Crystal Growth I, Springer, Berlin, 1979.

[12] F. Rosenberger, G. Müller, Interfacial transport in crystal growth, a parametric comparison of convective effects, J. Crystal Growth 65 (1983) 91104.

[13] R. Balasubramanian, S. Ostrach, Fluid motion in the Czochralski method of crystal growth, PhysicoChem. Hydrodyn. 5 (1984) 3-18.

[14] J. J. Derby, Theoretical modeling of Czochralski crystal growth, MRS Bulletin XIII (1988) 29-35.

[15] L. Hjellming, J. Walker, Melt motion in a Czochralski crystal puller with an axial magnetic field: Motion due to buoyancy and thermocapillarity, J. Fluid Mech 182 (1987) 335-368.

[16] A. D. W. Jones, Scaling analysis of the flow of a low Prandtl number Czochralski melt, J. Crystal Growth 88 (1988) 465-476.

[17] A. A. Wheeler, Boundary layer models in Czochralski crystal growth, J. Crystal Growth 97 (1989) 64-75.

[18] P. A. Sackinger, R. A. Brown, J. J. Derby, A finite-element method for analysis of fluid flow, heat transfer and free interfaces in Czochralski crystal growth, Int. J. Numer. Meth. Fluids 9 (1989) 453-492.

[19] D. Schwabe, Surface-tension-driven flow in crystal growth melts, in: Superhard Materials, Convection, and Optical Devices, Vol. 11 of Crystals, Springer, 1988, pp. $75-112$.

[20] H. C. Kuhlmann, Thermocapillary Convection in Models of Crystal Growth, Springer, 1999.

[21] O. Reynolds, An experimental investigation of the circumstances which determine whether the motion of water shall be direct or sinuous, and of the law of resistance in parallel channels, Phil. Trans. R. Soc. Lond. 174 (1883) 935-982.

[22] L. Rayleigh, On convective currents in a horizontal layer of fluid when the higher temperature is on the under side, Philos. Mag. 32 (1916) 475-480.

[23] H. Bénard, Les tourbillons cellulaires dans une nappe liquide transportant de la chaleur par convection en régime permanent, Annales de Chimie et de Physique 23 (1901) 62-144.

[24] W. R. Wilcox, Influence of convection on the growth of crystals from solution, J. Crystal Growth 65 (1983) 133-142. 
[25] H. J. Scheel, E. O. Schulz-Dubois, Flux growth of large crystals by accelerated crucible-rotation technique, J. Crystal Growth 8 (1971) 304-306.

[26] E. O. Schulz-Dubois, Accelerated crucible rotation: Hydrodynamics and stirring effect, J. Crystal Growth 12 (1972) 81-87.

[27] D. Elwell, H. J. Scheel, Crystal Growth from High-Temperature Solutions, Academic Press, 1975.

[28] R. Janssen-van Rosmalen, P. Bennema, Simulation of perturbations in trains of steps on crystal surfaces, J. Crystal Growth 32 (1976) 293-302.

[29] W. J. P. van Enckevort, R. Janssen-van Rosmalen, H. Klapper, W. H. van der Linden, Growth phenomena of KDP crystals in relation to the internal structure, J. Crystal Growth 60 (1982) 67-78.

[30] P. F. Bordui, J. C. Jacco, G. M. Loiacono, R. A. Stolzenberger, J. J. Zola, Growth of large single crystals of $\mathrm{KTiOPO}_{4}(\mathrm{KTP})$ from high-temperature solution using heat pipe based furnace system, J. Crystal Growth 84 (1987) 403-408.

[31] P. F. Bordui, S. Motakef, Hydrodynamic control of solution inclusion during crystal growth of $\mathrm{KTiOPO}_{4}$ (KTP) from high-temperature solution, J. Crystal Growth 96 (1989) 405-412.

[32] P. F. Bordui, Crystal growth of KTP from high-temperature solution, Ph.D. thesis, MIT (1987).

[33] D. Maynes, J. Klewicki, P. McMurtry, H. Robey, Hydrodynamic scalings in the rapid growth of crystals from solution, Journal of Crystal Growth 178 (4) (1997) 545-558.

[34] Y. Zhou, J. J. Derby, Three-dimensional computations of solution hydrodynamics during growth of potassium dihydrogen phosphate: I. Spin up and steady rotation, J. Crystal Growth 180 (1997) 497-509.

[35] A. Yeckel, Y. Zhou, M. Dennis, J. J. Derby, Three-dimensional computations of solution hydrodynamics during the growth of potassium dihydrogen phosphate: II. Spin down, J. Crystal Growth 191 (1998) 206-224.

[36] J. J. Derby, Y. I. Kwon, J. C. Rojo, B. Vartak, A. Yeckel, Finite element modeling of 3D fluid dynamics in crystal growth systems, Int. J. Comput. Fluid Dyn. 12 (1999) 225-240.

[37] B. Vartak, Y. I. Kwon, A. Yeckel, J. J. Derby, An analysis of flow and mass transfer during the solution growth of potassium titanyl phosphate, J. Crystal Growth 210 (2000) 704-718.

[38] B. Vartak, J. J. Derby, On stable algorithms and accurate solutions for convection-dominated mass transfer in crystal growth modeling, J. Crystal Growth 230 (2001) 202-209. 
[39] Y. I. Kwon, J. J. Derby, Modeling the coupled effects of interfacial and bulk phenomena during solution crystal growth, J. Crystal Growth 230 (2001) $328-335$.

[40] B. Vartak, A. Yeckel, J. J. Derby, Time-dependent, three-dimensional flow and mass transport during solution growth of potassium titanyl phosphate, J. Crystal Growth 281 (2005) 391-406.

[41] B. Vartak, A. Yeckel, J. J. Derby, On the validity of boundary layer analysis for flow and mass transfer caused by rotation during the solution growth of large, single crystals, J. Crystal Growth 283 (2005) 479-489.

[42] D. Gasperino, A. Yeckel, B. K. Olmstead, M. D. Ward, J. J. Derby, Mass transfer limitations at crystallizing interfaces in an atomic force microscopy fluid cell: A finite element analysis, Langmuir 22 (15) (2006) 6578-6586.

[43] Y. I. Kwon, B. Dai, J. J. Derby, Assessing the dynamics of liquid-phase solution growth via step growth models: From BCF to FEM, Prog. Crystal Growth and Charact. 53 (2007) 167-206.

[44] M. Sugiyama, D. Gasperino, J. J. Derby, V. H. Barocas, Protein-saltwater solution phase diagram determination by a combined experimentalcomputational scheme, Crystal Growth \& Design 8 (2008) 4208-4214.

[45] P. Rudolph, Travelling magnetic fields applied to bulk crystal growth from the melt: The step from basic research to industrial scale, J. Crystal Growth 310 (2008) 1298-1306.

[46] N. Ono, G. Trapaga, A numerical study of the effects of electromagnetic stirring on the distributions of temperature and oxygen concentration in silicon double-crucible Czochralski processing, J. Electrochem. Soc. 144 (2009) 764-772.

[47] J. Donea, S. Giuliani, A. Philippe, Finite elements in the solution of electromagnetic induction problems, Int. J. Num. Methods Eng. 8 (1974) 359-367.

[48] P. M. Gresho, J. J. Derby, A finite element model for induction heating of a metal crucible, J. Crystal Growth 85 (1987) 40-48.

[49] S. Yesilyurt, S. Motakef, R. Grugel, K. Mazuruk, The effect of the traveling magnetic field (TMF) on the buoyancy-induced convection in the vertical Bridgman growth of semiconductors, J. Crystal Growth 263 (2004) 80-89.

[50] P. Schwesig, M. Hainke, J. Friedrich, G. Müller, Comparative numerical study of the effects of rotating and travelling magnetic fields on the interface shape and thermal stress in the VGF growth of InP crystals, J. Crystal Growth 266 (2004) 224-228.

[51] I. Grants, G. Gerbeth, Stability of melt flow due to a traveling magnetic field in a closed ampoule, J. Crystal Growth 269 (2004) 630-638. 
[52] V. Galindo, I. Grants, R. Lantzsch, O. Pätzold, G. Gerbeth, Numerical and experimental modeling of the melt flow in a traveling magnetic field for vertical gradient freeze crystal growth, J. Crystal Growth 303 (1) (2007) $258-261$.

[53] R. Lantzsch, V. Galindo, I. Grants, C. Zhang, O. Pätzold, G. Gerbeth, M. Stelter, Experimental and numerical results on the fluid flow driven by a traveling magnetic field, J. Crystal Growth 305 (1) (2007) 249-256.

[54] K. Niemietz, V. Galindo, O. Pätzold, G. Gerbeth, M. Stelter, Flow modelling with relevance to vertical gradient freeze crystal growth under the influence of a travelling magnetic field, J. Crystal Growth 318 (2011) 150155.

[55] V. Galindo, K. Niemietz, O. Pätzold, G. Gerbeth, Numerical and experimental modeling of VGF-type buoyant flow under the influence of traveling and rotating magnetic fields, J. Crystal Growth 360 (2012) 30-34.

[56] C. Frank-Rotsch, D. Jockel, M. Ziem, P. Rudolph, Numerical optimization of the interface shape at the VGF growth of semiconductor crystals in a traveling magnetic field, J. Crystal Growth 310 (2008) 1505-1510.

[57] C. Frank-Rotsch, P. Rudolph, Vertical gradient freeze of 4 inch Ge crystals in a heater-magnet module, J. Crystal Growth 311 (2009) 2294-2299.

[58] N. Dropka, W. Miller, R. Menzel, U. Rehse, Numerical study on transport phenomena in a directional solidification process in the presence of travelling magnetic fields, J. Crystal Growth 312 (2010) 1407-1410.

[59] N. Dropka, W. Miller, U. Rehse, P. Rudolph, F. Büllesfeld, U. Sahr, O. Klein, D. Reinhardt, Numerical study on improved mixing in silicon melts by double-frequency TMF, J. Crystal Growth 318 (2011) 275-279.

[60] N. Dropka, C. Frank-Rotsch, W. Miller, P. Rudolph, Influence of travelling magnetic fields on S-L interface shapes of materials with different electrical conductivities, J. Crystal Growth 338 (1) (2012) 208-213.

[61] N. Dropka, C. Frank-Rotsch, P. Rudolph, Comparison of stirring efficiency of various non-steady magnetic fields during unidirectional solidification of large silicon melts, Journal of Crystal Growth 365 (2013) 64-72.

[62] N. Dropka, C. Frank-Rotsch, Accelerated VGF-crystal growth of GaAs under traveling magnetic fields, Journal of Crystal Growth 367 (2013) 1-7.

[63] C. Frank-Rotsch, N. Dropka, A. Glacki, U. Juda, VGF growth of GaAs utilizing heater-magnet module, Journal of Crystal Growth 401 (2014) 702707.

[64] N. Dropka, C. Frank-Rotsch, Enhanced VGF-GaAs growth using pulsed unidirectional TMF, Journal of Crystal Growth 386 (2014) 146-153. 
[65] A. Yeckel, J. J. Derby, The prospects for traveling magnetic fields to affect interface shape in the vertical gradient freeze growth of cadmium zinc telluride, J. Crystal Growth 364 (2013) 133-144.

[66] S. Wang, H. Fang, Z. Jin, C. Zhao, L. Zheng, Integrated analysis and design optimization of germanium purification process using zone-refining technique, Journal of Crystal Growth 408 (2014) 42-48.

[67] T. Jasinski, A. F. Witt, On control of the crystal melt interface shape during growth in a vertical Bridgman configuration, J. Crystal Growth 71 (1985) 295-304.

[68] W. A. Tiller, Principles of solidification, in: J. J. Gilman (Ed.), The Art and Science of Growing Crystals, John Wiley \& Sons, Inc., 1963, p. 276.

[69] J. C. Brice, Crystal Growth Processes, Halsted Press, New York, 1986.

[70] T. P. Lyubimova, A. Croell, P. Dold, O. A. Khlybov, I. S. Fayzrakhmanova, Time-dependent magnetic field influence on GaAs crystal growth by vertical Bridgman method, J. Crystal Growth 266 (2004) 404-410.

[71] P. Rudolph, M. Mühlberg, Basic problems of vertical Bridgman growth of CdTe, Mater. Sci. Eng. B 16 (1993) 8-16.

[72] P. Rudolph, Fundamental studies on Bridgman growth of CdTe, Progress in Crystal Growth and Characterization of Materials 29 (1994) 275-281.

[73] N. Zhang, A. Yeckel, J. J. Derby, Maintaining convex interface shapes during electrodynamic gradient freeze growth of cadmium zinc telluride using a dynamic, bell-curve furnace profile, J. Crystal Growth 355 (2012) $113-121$.

[74] C. Belouet, M. Monnier, Autoradiography as a tool for studying iron segregation and related defects in KDP single crystals, J. Crystal Growth 29 (1975) 109-120.

[75] C. Belouet, Growth and characterization of single crystals of KDP family, Prog. Crystal Growth and Charact. 3 (1981) 121-156.

[76] A. A. Chernov, Stability of faceted shapes, J. Crystal Growth 24/25 (1974) $11-31$.

[77] A. A. Chernov, How does the flow within the boundary layer influence morphological stability of a vicinal face?, J. Crystal Growth 118 (1992) 333-347.

[78] A. A. Chernov, S. R. Coriell, B. T. Murray, Morphological stability of a vicinal face induced by step flow, J. Crystal Growth 132 (1993) 405-413. 
[79] S. R. Coriell, B. T. Murray, A. A. Chernov, G. B. McFadden, Step bunching on a vicinal face of a crystal growing in a flowing solution, J. Crystal Growth 169 (1996) 773-785.

[80] S. Y. Potapenko, Morphological instability of steps during crystal growth from solution flow, J. Crystal Growth 158 (1996) 346-358.

[81] H. Lin, R. G. Vekilov, F. Rosenberger, Facet morphology response to nonuniformities in nutrient and impurity supply. II. Numerical simulations, J. Crystal Growth 158 (1996) 552-559.

[82] P. G. Vekilov, J. I. D. Alexander, F. Rosenberger, Nonlinear response of layer growth dynamics in the mixed kinetics-bulk-transport regime, Phys. Rev. E 54 (6) (1996) 6650-6660.

[83] P. G. Vekilov, H. Lin, F. Rosenberger, Unsteady crystal growth due to step-bunch cascading, Phys. Rev. E 55 (3) (1997) 3202-3214.

[84] F. Rosenberger, H. Lin, P. G. Vekilov, Finite-amplitude instability in growth step trains with overlapping step supply fields, Phys. Rev. E 59 (3) (1999) 3155-3164.

[85] M. Funaki, T. Ozaki, K. Satoh, R. Ohno, Growth and characterization of CdTe single crystals for radiation detectors, Nuclear Instruments and Methods in Physics Research Section A: Accelerators, Spectrometers, Detectors and Associated Equipment 436 (1999) 120-126.

[86] H. Chen, S. Awadalla, J. Mackenzie, R. Redden, G. Bindley, A. Bolotnikov, G. Camarda, G. Carini, R. James, Characterization of traveling heater method (THM) grown $\mathrm{Cd}_{0.9} \mathrm{Zn}_{0.1} \mathrm{Te}$ crystals, IEEE Transactions on Nuclear Science, 54 (4) (2007) 811-816.

[87] S. Awadalla, J. Mackenzie, H. Chen, B. Redden, G. Bindley, M. Duff, A. Burger, M. Groza, V. Buliga, J. Bradley, Z. Dai, N. Teslich, D. Black, Characterization of detector-grade CdZnTe crystals grown by traveling heater method (THM), Journal of Crystal Growth 312 (4) (2010) 507-513.

[88] A. El Mokri, R. Triboulet, A. Lusson, A. Tromson-Carli, G. Didier, Growth of large, high purity, low cost, uniform CdZnTe crystals by the "cold travelling heater method", J. Crystal Growth 138 (4) (1994) 168-174.

[89] Y. Wang, K. Kudo, Y. Inatomi, R. Ji, T. Motegi, Growth interface of CdZnTe grown from Te solution with THM technique under static magnetic field, Journal of Crystal Growth 284 (2005) 406-411.

[90] C. W. Lan, D. T. Yang, A computer simulation of crystal growth by the traveling-solvent method (TSM): Pseudo-steady-state calculations, Modelling and Simulation in Materials Science and Engineering 3 (1) (1995) 71-92. 
[91] C. Stelian, T. Duffar, Numerical modeling of CdTe crystallization from Te solution under terrestrial and microgravity conditions, Journal of Crystal Growth 400 (2014) 67-75.

[92] J. H. Peterson, A. Yeckel, J. J. Derby, A fundamental limitation on growth rates in the traveling heater method, J. Crystal Growth (2016) in press.

[93] T. H. Bell, Lee waves in stratified flows with simple harmonic time dependence, Journal of Fluid Mechanics 67 (1975) 705-722.

[94] C. J. Nappo, Fundamentals, in: C. J. Nappo (Ed.), An Introduction to Atmospheric Gravity Waves, Vol. 102 of International Geophysics, Academic Press, 2012, pp. 29-56.

[95] W. W. Mullins, R. F. Sekerka, Stability of a planar interface during solidification of a dilute binary alloy, J. Appl. Phys. 35 (1964) 444-451.

[96] J. Kim, P. Moin, Tackling turbulence with supercomputers, Scientific American (1997) 62-68.

[97] K. M. Kim, A. F. Witt, H. C. Gatos, Crystal growth from the melt under destabilizing thermal gradients, J. Electrochem Soc. 119 (1972) 1218-1226.

[98] K. M. Kim, A. F. Witt, M. Lichtensteiger, H. C. Gatos, Quantitative analysis of the effects of destabilizing vertical thermal gradients on crystal growth and segregation: Ga-doped Ge, J. Electrochem. Soc. 125 (1978) 475-480. 